\title{
Global Existence and Asymptotic Behavior of Solutions to the Generalized Damped Boussinesq Equation
}

\author{
Yinxia Wang ${ }^{1}$ and Hengjun Zhao ${ }^{2}$ \\ ${ }^{1}$ School of Mathematics and Information Sciences, North China University of Water Resources and Electric Power, \\ Zhengzhou 450011, China \\ ${ }^{2}$ College of Science, Henan Institute of Engineering, Zhengzhou 450001, China
}

Correspondence should be addressed to Yinxia Wang; yinxial17@126.com

Received 26 January 2013; Accepted 12 July 2013

Academic Editor: B. G. Konopelchenko

Copyright (c) 2013 Y. Wang and H. Zhao. This is an open access article distributed under the Creative Commons Attribution License, which permits unrestricted use, distribution, and reproduction in any medium, provided the original work is properly cited.

We investigate the Cauchy problem for the generalized damped Boussinesq equation. Under small condition on the initial value, we prove the global existence and optimal decay estimate of solutions for all space dimensions $n \geq 1$. Moreover, when $n \geq 2$, we show that the solution can be approximated by the linear solution as time tends to infinity.

\section{Introduction}

We investigate the Cauchy problem of the following generalized damped Boussinesq equation:

$$
u_{t t}-\Delta u-2 b \Delta u_{t}+\alpha \Delta^{2} u=\Delta f(u)
$$

with the initial value

$$
t=0: u=u_{0}(x), \quad u_{t}=u_{1}(x) .
$$

Here $u=u(x, t)$ is the unknown function of $x=\left(x_{1}, \ldots\right.$, $\left.x_{n}\right) \in \mathbb{R}^{n}$ and $t>0, b>0$, and $\alpha>0$ are constants. The nonlinear term $f(u)$ is a given smooth function of $u \in \mathbb{R}$ satisfying $f(u)=O\left(u^{2}\right)$ for $u \rightarrow 0$.

It is well known that the classical Boussinesq equation

$$
u_{t t}+\alpha \Delta^{2} u-\Delta u=\beta \Delta\left(u^{2}\right)
$$

was derived by Boussinesq [1] in 1872 to describe shallow water waves, where $u(x, t)$ is an elevation of the free surface of fluid and the constant coefficients $\alpha$ and $\beta$ depend on the depth of fluid and the characteristic speed of long waves. It is interesting to note that this equation also governs nonlinear string oscillations.
Taking into account dispersion and nonlinearity, but in real processes viscosity also plays an important role. Varlamov considered the following damped Boussinesq equation (see [2-4]):

$$
u_{t t}-\Delta u-2 b \Delta u_{t}+\alpha \Delta^{2} u=\beta \Delta\left(u^{2}\right),
$$

where $b>0$ and $\alpha>0$ are constants. Under small condition on the initial value, Varlamov [2] obtained a classical solution to the problem (4), (2) by means of the application of both the spectral and perturbation theories. Moreover, large time asymptotics of this solution was also discussed. For the problem (4), (2) in one, two, and three space dimensions, existence and uniqueness of local solution are proved by Varlamov [3]. The author also showed that for discontinuous initial perturbations this solution is infinitely differentiable with respect to time $t$ and space coordinates for $t>0$ on a bounded time interval. Existence and uniqueness of the classical solution for the problem (4), (2) in two space dimensions was proved, and the solution was constructed in the form of a series. The major term of its long-time asymptotics is calculated explicitly, and a uniform in space estimate of the residual term was given (see [4]). 
The main purpose of this paper is to establish the following optimal decay estimate of solutions to (1), (2) for $n \geq 1$ :

$$
\left\|\partial_{x}^{k} u(t)\right\|_{H^{s+2-k}} \leq C E_{0}(1+t)^{-n / 4-k / 2}
$$

for $0 \leq k \leq s+2$ and $s \geq \max \{0,[n / 2]-1\}$. Here $E_{0}:=$ $\left\|u_{0}\right\|_{L^{1}}+\left\|u_{1}\right\|_{\dot{W}^{-1,1}}+\left\|u_{0}\right\|_{H^{s+2}}+\left\|u_{1}\right\|_{H^{s}}$ is assumed to be small. Moreover, when $n \geq 2$, we show that our solution $u$ can be approximated by the solution $u_{L}$ to the linearized problem, namely, the problem (1), (2) with $f(u) \equiv 0$. More precisely, when $n \geq 2$, we show that

$$
\left\|\partial_{x}^{k}\left(u-u_{L}\right)(t)\right\|_{H^{s+2-k}} \leq C E_{0}^{2}(1+t)^{-n / 4-k / 2} \eta(t)
$$

for $0 \leq k \leq s+2$ and $s \geq \max \{0,[n / 2]-1\}$, where $\eta(t)=$ $(1+t)^{-1 / 2} \log (2+t)$ for $n=2$ and $\eta(t)=(1+t)^{-1 / 2}$ for $n \geq 3$.

The study of the global existence and asymptotic behavior of solutions to hyperbolic-type equations has a long history. We refer to [5, 6] for hyperbolic equations, [7-10] for damped wave equation and [11-17] for various aspects of dissipation of the plate equation.

The paper is organized as follows. In Section 2, we derive the solution formula of our semilinear problem. We study the decay property of the solution operators appearing in the solution formula in Section 3. Then, in Section 4, we prove the global existence and asymptotic decay of solutions. Finally, we derive a simpler asymptotic profile which gives the approximation to the linear solution in Section 5.

Notations. We give some notations which are used in this paper. Let $\mathscr{F}[u]$ denote the Fourier transform of $u$ defined by

$$
\widehat{u}(\xi)=\mathscr{F}[u]=\int_{\mathbb{R}^{n}} e^{-i \xi \cdot x} u(x) d x,
$$

and we denote its inverse transform by $\mathscr{F}^{-1}$.

For $1 \leq p \leq \infty, L^{p}=L^{p}\left(\mathbb{R}^{n}\right)$ denotes the usual Lebesgue space with the norm $\|\cdot\|_{L^{p}}$. The usual Sobolev space of $s$ is defined by $W^{s, p}=(I-\Delta)^{-s / 2} L^{p}$ with the norm $\|f\|_{W^{s, p}}=$ $\left\|(I-\Delta)^{s / 2} f\right\|_{L^{p}}$; the homogeneous Sobolev space of $s$ is defined by $\dot{W}^{s, p}=(-\Delta)^{-s / 2} L^{p}$ with the norm $\|f\|_{\dot{W}^{s, p}}=$ $\left\|(-\Delta)^{s / 2} f\right\|_{L^{p}}$; especially $H^{s}=W^{s, 2}, \dot{H}^{s}=\dot{W}^{s, 2}$. Moreover, we know that $W^{s, p}=L^{p} \bigcap \dot{W}^{s, p}$ for $s \geq 0$.

Finally, in this paper, we denote every positive constant by the same symbol $C$ or $c$ without confusion. $[\cdot]$ is the Gauss symbol.

\section{Solution Formula}

The aim of this section is to derive the solution formula for the problem (1), (2). We first investigate the linearized equation of (1):

$$
u_{t t}-\Delta u-2 b \Delta u_{t}+\alpha \Delta^{2} u=0
$$

with the initial data in (2). We apply the Fourier transform to (8). This yields

$$
\widehat{u}_{t t}+2 b|\xi|^{2} \widehat{u}_{t}+\left(|\xi|^{2}+\alpha|\xi|^{4}\right) \widehat{u}=0 .
$$

The corresponding initial value is given as

$$
t=0: \widehat{u}=\widehat{u}_{0}(\xi), \quad \widehat{u}_{t}=\widehat{u}_{1}(\xi)
$$

The characteristic equation of (9) is

$$
\lambda^{2}+2 b|\xi|^{2} \lambda+|\xi|^{2}+\alpha|\xi|^{4}=0
$$

Let $\lambda=\lambda_{ \pm}(\xi)$ be the corresponding eigenvalues of (11), and we obtain

$$
\lambda_{ \pm}(\xi)=-b|\xi|^{2} \pm|\xi| \sqrt{\left(b^{2}-\alpha\right)|\xi|^{2}-1}
$$

The solution to the problem (9), (10) in the Fourier space is then given explicitly in the form

$$
\widehat{u}(\xi, t)=\widehat{G}(\xi, t) \widehat{u}_{1}(\xi)+\widehat{H}(\xi, t) \widehat{u}_{0}(\xi),
$$

where

$$
\begin{gathered}
\widehat{G}(\xi, t)=\frac{1}{\lambda_{+}(\xi)-\lambda_{-}(\xi)}\left(e^{\lambda_{+}(\xi) t}-e^{\lambda_{-}(\xi) t}\right) \\
\widehat{H}(\xi, t)=\frac{1}{\lambda_{+}(\xi)-\lambda_{-}(\xi)}\left(\lambda_{+}(\xi) e^{\lambda_{-}(\xi) t}-\lambda_{-}(\xi) e^{\lambda_{+}(\xi) t}\right) .
\end{gathered}
$$

We define $G(x, t)$ and $H(x, t)$ by

$$
\begin{aligned}
G(x, t) & =\mathscr{F}^{-1}[\widehat{G}(\xi, t)](x), \\
H(x, t) & =\mathscr{F}^{-1}[\widehat{H}(\xi, t)](x),
\end{aligned}
$$

respectively, where $\mathscr{F}^{-1}$ denotes the inverse Fourier transform. Then, applying $\mathscr{F}^{-1}$ to (13), we obtain

$$
u(t)=G(t) * u_{1}+H(t) * u_{0} .
$$

By the Duhamel principle, we obtain the solution formula to (1), (2) as follows:

$$
u(t)=G(t) * u_{1}+H(t) * u_{0}+\int_{0}^{t} G(t-\tau) * \Delta f(u)(\tau) d \tau
$$

\section{Decay Property}

The aim of this section is to establish decay estimates of the solution operators $G(t)$ and $H(t)$ appearing in (16) and (17), respectively.

Lemma 1. The solution of the problem (9), (10) satisfies

$$
\begin{aligned}
& \left(|\xi|^{2}+|\xi|^{4}\right)|\widehat{u}(\xi, t)|^{2}+\left|\widehat{u}_{t}(\xi, t)\right|^{2} \\
& \quad \leq C e^{-c|\xi|^{2} t}\left(\left(|\xi|^{2}+|\xi|^{4}\right)\left|\widehat{u}_{0}(\xi)\right|^{2}+\left|\widehat{u}_{1}(\xi)\right|^{2}\right)
\end{aligned}
$$

for $\xi \in \mathbb{R}^{n}$ and $t \geq 0$. 
Proof. Multiplying (9) by $\overline{\widehat{u}}_{t}$ and taking the real part yield

$$
\frac{1}{2} \frac{d}{d t}\left\{\left|\widehat{u}_{t}\right|^{2}+|\xi|^{2}|\widehat{u}|^{2}+\alpha|\xi|^{4}|\widehat{u}|^{2}\right\}+2 b|\xi|^{2}\left|\widehat{u}_{t}\right|^{2}=0 .
$$

Multiplying (9) by $\overline{\widehat{u}}$ and taking the real part, we obtain

$$
\begin{aligned}
& \frac{1}{2} \frac{d}{d t}\left\{2 b|\xi|^{2}|\widehat{u}|^{2}+2 \operatorname{Re}\left(\widehat{u}_{t} \overline{\hat{u}}\right)\right\} \\
& \quad+|\xi|^{2}|\widehat{u}|^{2}+\alpha|\xi|^{4}|\widehat{u}|^{2}-\left|\widehat{u}_{t}\right|^{2}=0 .
\end{aligned}
$$

Multiplying both sides of (22) by $b|\xi|^{2}$ and summing up the resulting equation and (21) yield

$$
\frac{d}{d t} E+F=0,
$$

where

$$
\begin{gathered}
E=\frac{1}{2}\left|\widehat{u}_{t}\right|^{2}+\frac{1}{2}|\xi|^{2}|\widehat{u}|^{2}+\frac{\alpha}{2}|\xi|^{4}|\widehat{u}|^{2} \\
+b^{2}|\xi|^{4}|\widehat{u}|^{2}+b|\xi|^{2} \operatorname{Re}\left(\widehat{u}_{t} \overline{\hat{u}}\right), \\
F=b|\xi|^{2}\left|\widehat{u}_{t}\right|^{2}+b|\xi|^{4}|\widehat{u}|^{2}+b \alpha|\xi|^{6}|\widehat{u}|^{2} .
\end{gathered}
$$

A simple computation implies that

$$
C E_{0} \leq E \leq C E_{0},
$$

where

$$
E_{0}=\left(|\xi|^{2}+|\xi|^{4}\right)|\widehat{u}|^{2}+\left|\widehat{u}_{t}\right|^{2}
$$

Note that

$$
F \geq c|\xi|^{2} E_{0} .
$$

It follows from (25) that

$$
F \geq c|\xi|^{2} E .
$$

Using (23) and (28), we get

$$
\frac{d}{d t} E+c|\xi|^{2} E \leq 0 .
$$

Thus

$$
E(\xi, t) \leq e^{-c|\xi|^{2} t} E(\xi, 0),
$$

which together with (25) proves the desired estimates (20). Then we have completed the proof of the lemma.

Lemma 2. Let $\widehat{G}(\xi, t)$ and $\widehat{H}(\xi, t)$ be the fundamental solution of (8) in the Fourier space, which are given in (14) and (15), respectively. Then one has the estimates

$$
\begin{gathered}
\left(|\xi|^{2}+|\xi|^{4}\right)|\widehat{G}(\xi, t)|^{2}+\left|\widehat{G}_{t}(\xi, t)\right|^{2} \leq C e^{-c|\xi|^{2} t} \\
\left(|\xi|^{2}+|\xi|^{4}\right)|\widehat{H}(\xi, t)|^{2}+\left|\widehat{H}_{t}(\xi, t)\right|^{2} \leq C\left(|\xi|^{2}+|\xi|^{4}\right) e^{-c|\xi|^{2} t}
\end{gathered}
$$

for $\xi \in \mathbb{R}^{n}$ and $t \geq 0$.
Proof. Firstly, we investigate the problem (8), (2) with $\widehat{u}_{0}(\xi)=$ 0 ; from (13), we obtain

$$
\widehat{u}(\xi, t)=\widehat{G}(\xi, t) \widehat{u}_{1}(\xi), \quad \widehat{u}_{t}(\xi, t)=\widehat{G}_{t}(\xi, t) \widehat{u}_{1}(\xi) .
$$

Substituting the equalities into (20) with $\widehat{u}_{0}(\xi)=0$, we get (31).

In what follows, we consider the problem (8), (2) with $\widehat{u}_{1}(\xi)=0$; it follows from (13) that

$$
\widehat{u}(\xi, t)=\widehat{H}(\xi, t) \widehat{u}_{0}(\xi), \quad \widehat{u}_{t}(\xi, t)=\widehat{H}_{t}(\xi, t) \widehat{u}_{0}(\xi) .
$$

Substituting the equalities into $(20)$ with $\widehat{u}_{1}(\xi)=0$, we get the desired estimate (32). The lemma is proved.

Lemma 3. Let $G(x, t)$ and $H(x, t)$ be the fundamental solution of (8), which are given in (16) and (17), respectively. Let $1 \leq p \leq$ 2 , and let $k, j$, and $l$ be nonnegative integers. Then one has

$$
\begin{aligned}
& \left\|\partial_{x}^{k} G(t) * \phi\right\|_{L^{2}} \\
& \leq C(1+t)^{-(n / 2)(1 / p-1 / 2)-(k-j) / 2}\left\|\partial_{x}^{j} \phi\right\|_{W^{-1, p}} \\
& \quad+C e^{-c t}\left\|\partial_{x}^{k+l-2} \phi\right\|_{L^{2}}, \\
& \left\|\partial_{x}^{k} H(t) * \phi\right\|_{L^{2}} \\
& \leq C(1+t)^{-(n / 2)(1 / p-1 / 2)-(k-j) / 2}\left\|\partial_{x}^{j} \phi\right\|_{L^{p}} \\
& \quad+C e^{-c t}\left\|\partial_{x}^{k+l} \phi\right\|_{L^{2}}
\end{aligned}
$$

for $0 \leq j \leq k$, where $k+l-2 \geq 0$ in (35). Similarly, one has

$$
\begin{aligned}
\left\|\partial_{x}^{k} G_{t}(t) * \phi\right\|_{L^{2}} & \\
\leq & C(1+t)^{-(n / 2)(1 / p-1 / 2)-(k+1-j) / 2}\left\|\partial_{x}^{j} \phi\right\|_{\dot{W}^{-1, p}} \\
& +C e^{-c t}\left\|\partial_{x}^{k+l} \phi\right\|_{L^{2}} \\
\left\|\partial_{x}^{k} H_{t}(t) * \phi\right\|_{L^{2}} & \\
\leq & C(1+t)^{-(n / 2)(1 / p-1 / 2)-(k+1-j) / 2}\left\|\partial_{x}^{j} \phi\right\|_{L^{p}} \\
& +C e^{-c t}\left\|\partial_{x}^{k+l+2} \phi\right\|_{L^{2}}
\end{aligned}
$$

for $0 \leq j \leq k+1$.

Proof. We only prove (35). By the Plancherel theorem, (31), and Hausdorff-Young inequality, we obtain

$$
\begin{aligned}
\left\|\partial_{x}^{k} G(t) * \phi\right\|_{L^{2}}^{2}= & \int_{|\xi| \leq 1}|\xi|^{2|k|}|\widehat{G}(\xi, t)|^{2}|\widehat{\phi}(\xi)|^{2} d \xi \\
& +\int_{|\xi| \geq 1}|\xi|^{2|k|}|\widehat{G}(\xi, t)|^{2}|\widehat{\phi}(\xi)|^{2} d \xi \\
\leq & C \int_{|\xi| \leq 1}|\xi|^{2|k|-2} e^{-c|\xi|^{2} t}|\widehat{\phi}(\xi)|^{2} d \xi \\
& +C e^{-c t} \int_{|\xi| \geq 1}|\xi|^{2 k}\left(|\xi|^{2}+|\xi|^{4}\right)^{-2}|\widehat{\phi}(\xi)|^{2} d \xi \\
:= & \mathscr{A}_{1}+\mathscr{A}_{2} .
\end{aligned}
$$


For the term $\mathscr{A}_{1}$, letting $1 / p^{\prime}+1 / p=1$, we have

$$
\begin{aligned}
\mathscr{A}_{1} & \leq C \int_{|\xi| \leq 1}|\xi|^{2 k-2} e^{-c|\xi|^{2} t}|\widehat{\phi}(\xi)|^{2} d \xi \\
& \leq C\left\||\xi|^{j-1} \hat{\phi}\right\|_{L^{p^{\prime}}}^{2}\left(\int_{|\xi| \leq 1}|\xi|^{2(k-j) p} e^{-c q|\xi|^{2} t} d \xi\right)^{1 / p} \\
& \leq C(1+t)^{-n(1 / p-1 / 2)-(k-j)}\left\|\partial_{x}^{j} \phi\right\|_{\dot{W}^{-1, p}}^{2},
\end{aligned}
$$

where we used the Hölder inequality with $2 / p^{\prime}+1 / q=1$ and the Hausdorff-Young inequality $\|\widehat{v}\|_{L^{p^{\prime}}} \leq C\|v\|_{L^{p}}$ for $v=$ $(-\Delta)^{-1 / 2} \partial_{x}^{j} \phi$. On the other hand, we can estimate the term $\mathscr{A}_{2}$ simply as

$$
\begin{aligned}
\mathscr{A}_{2} & \leq C e^{-c t} \int_{|\xi| \geq 1}|\xi|^{2 k-4}|\widehat{\phi}(\xi)|^{2} d \xi \\
& \leq C e^{-c t} \int_{|\xi| \geq 1}|\xi|^{2(k+l-2)}|\widehat{\phi}(\xi)|^{2} d \xi \\
& \leq C e^{-c t}\left\|\partial_{x}^{k+l-2} \phi\right\|_{L^{2}}^{2},
\end{aligned}
$$

where $k+l-2 \geq 0$.

Combining the previous three inequalities yields (35). This completes the proof of Lemma 3. lary.

From Lemma 3, we immediately have the following corol-

Corollary 4. Let $G(x, t)$ and $H(x, t)$ be the fundamental solution of (8), which are given in (15) and (16), respectively. Let $1 \leq p \leq 2$, and let $k, j$, and $l$ be nonnegative integers. Then one has

$$
\begin{aligned}
\left\|\partial_{x}^{k} G(t) * \Delta g\right\|_{L^{2}} & \\
\leq & C(1+t)^{-(n / 2)(1 / p-1 / 2)-(k+1-j) / 2}\left\|\partial_{x}^{j} g\right\|_{L^{p}} \\
& +C e^{-c t}\left\|\partial_{x}^{k+l} g\right\|_{L^{2}}
\end{aligned}
$$

for $0 \leq k \leq j+1$. Also one has

$$
\begin{aligned}
&\left\|\partial_{x}^{k} G_{t}(t) * \Delta g\right\|_{L^{2}} \\
& \leq C(1+t)^{-(n / 2)(1 / p-1 / 2)-(k+2-j) / 2}\left\|\partial_{x}^{j} g\right\|_{L^{p}} \\
& \quad+C e^{-c t}\left\|\partial_{x}^{k+l+2} g\right\|_{L^{2}}
\end{aligned}
$$

for $0 \leq k \leq j+2$.

\section{Global Existence and Asymptotic Behavior of Solutions to (1), (2)}

The purpose of this section is to prove global existence and asymptotic behavior of solutions to the Cauchy problem (1), (2). We need the following lemma, which come from [18] (see also [19]).
Lemma 5. Assume that $f=f(v)$ is a smooth function. Suppose that $f(v)=O\left(|v|^{1+\theta}\right)(\theta \geq 1$ is an integer $)$ when $|v| \leq v_{0}$. Then for integer $m \geq 0$, if $v \in W^{m, q}\left(\mathbb{R}^{n}\right) \bigcap L^{p}\left(\mathbb{R}^{n}\right) \bigcap L^{\infty}\left(\mathbb{R}^{n}\right)$ and $\|v\|_{L^{\infty}} \leq v_{0}$, then the following inequalities hold:

$$
\left\|\partial_{x}^{m} f(v)\right\|_{L^{r}} \leq C\|v\|_{L^{p}}\left\|\partial_{x}^{m} v\right\|_{L^{q}}\|v\|_{L^{\infty}}^{\theta-1},
$$

where $1 / r=1 / p+1 / q, 1 \leq p, q, r \leq+\infty$.

Theorem 6. Let $n \geq 1$ and $s \geq \max \{0,[n / 2]-1\}$. Suppose that $f(u)$ is smooth and satisfies $f(u)=O\left(u^{2}\right)$ for $u \rightarrow 0$. Assume that $u_{0} \in H^{s+2} \cap L^{1}, u_{1} \in H^{s} \cap \dot{W}^{-1,1}$. Put

$$
E_{0}:=\left\|u_{0}\right\|_{L^{1}}+\left\|u_{1}\right\|_{\dot{W}^{-1,1}}+\left\|u_{0}\right\|_{H^{s+2}}+\left\|u_{1}\right\|_{H^{s}} .
$$

Then there exists a positive constant $\delta_{1}$ such that if $E_{0} \leq \delta_{1}$, the Cauchy problem (1), (2) has a unique global solution $u(x, t)$ satisfying

$$
u \in C^{0}\left([0, \infty) ; H^{s+2}\left(\mathbb{R}^{n}\right)\right) \bigcap C^{1}\left([0, \infty) ; H^{s}\left(\mathbb{R}^{n}\right)\right) .
$$

Moreover, the solution satisfies the decay estimate

$$
\begin{gathered}
\left\|\partial_{x}^{k} u(t)\right\|_{L^{2}} \leq C E_{0}(1+t)^{-n / 4-k / 2}, \\
\left\|\partial_{x}^{l} u_{t}(t)\right\|_{L^{2}} \leq C E_{0}(1+t)^{-n / 4-(k+1) / 2},
\end{gathered}
$$

where $0 \leq k \leq s+2$ in (47) and $0 \leq k \leq s$ in (48).

Proof. The existence and uniqueness of small solutions can be proved by the contraction mapping principle. Here we only show the decay estimates (47) and (48) for the solution $u$ of (19) satisfying $\|u(t)\|_{L^{\infty}} \leq M_{0}$ with some $M_{0}$. To this end, we introduce the quantity

$$
M(t)=\sum_{k=0}^{s+1} \sup _{0 \leq \tau \leq t}(1+\tau)^{n / 4+k / 2}\left\|\partial_{x}^{k} u(\tau)\right\|_{L^{2}} .
$$

Here we note that

$$
\|u(t)\|_{L^{\infty}} \leq C M(t)(1+t)^{-n / 2},
$$

provided that $s \geq \max \{0,[n / 2]-1\}$. This follows from the Gagliardo-Nirenberg inequality $\|u\|_{L^{\infty}} \leq C\left\|\partial_{x}^{s_{0}} u\right\|_{L^{2}}^{\theta}\|u\|_{L^{2}}^{1-\theta}$ with $s_{0}=[n / 2]+1$ and $\theta=n / 2 s_{0}$ and the definition of $M(t)$ in (49).

Applying $\partial_{x}^{k}$ to (19) and taking the $L^{2}$ norm, we obtain

$$
\begin{aligned}
&\left\|\partial_{x}^{k} u(t)\right\|_{L^{2}} \leq\left\|\partial_{x}^{k} G(t) * u_{1}\right\|_{L^{2}}+\left\|\partial_{x}^{k} H(t) * u_{0}\right\|_{L^{2}} \\
&+\int_{0}^{t}\|G(t-\tau) * \Delta f(u(\tau))\|_{L^{2}} d \tau \\
&:=I_{1}+I_{2}+J .
\end{aligned}
$$

Firstly, we estimate $I_{1}$. We apply (35) with $p=1, j=0$, and $l=0(l=2$ for $k=0, l=1$ for $k=1)$. This yields

$$
\begin{aligned}
I_{1} & \leq C(1+t)^{-n / 4-k / 2}\left\|u_{1}\right\|_{\dot{W}^{-1,1}}+C e^{-c t}\left\|\partial_{x}^{(k-2)_{+}} u_{1}\right\|_{L^{2}} \\
& \leq C E_{0}(1+t)^{-n / 4-k / 2},
\end{aligned}
$$


where $(k-2)_{+}=\max \{k-2,0\}$. Similarly, applying (36) with $p=1, j=0$, and $l=0$ to the term $I_{2}$, we have

$$
\begin{aligned}
I_{2} \leq & C(1+t)^{-n / 4-k / 2}\left\|u_{0}\right\|_{L^{1}} \\
& +C e^{-c t}\left\|\partial_{x}^{k} u_{0}\right\|_{L^{2}} \leq C E_{0}(1+t)^{-n / 4-k / 2} .
\end{aligned}
$$

We estimate the nonlinear term $J$. We divide $J$ into two parts and write $J=J_{1}+J_{2}$, where $J_{1}$ and $J_{2}$ are corresponding to the time intervals $[0, t / 2]$ and $[t / 2, t]$, respectively. For the term $J_{1}$, we apply (42) with $p=1, j=0$, and $l=0$. This yields

$$
\begin{aligned}
J_{1} \leq & C \int_{0}^{t / 2}(1+t-\tau)^{-n / 4-(k+1) / 2}\|f(u)(\tau)\|_{L^{1}} d \tau \\
& +C \int_{0}^{t / 2} e^{-c(t-\tau)}\left\|\partial_{x}^{k} f(u)(\tau)\right\|_{L^{2}} d \tau \\
& :=J_{11}+J_{12} .
\end{aligned}
$$

Here we see that $\|f(u)\|_{L^{1}} \leq C\|u\|_{L^{2}}^{2}$ by Lemma 5. Thus we have $\|f(u)(\tau)\|_{L^{1}} \leq C M(t)^{2}(1+\tau)^{-n / 2}$. Therefore we can estimate the term $J_{11}$ as

$$
\begin{aligned}
J_{11} & \leq C M(t)^{2} \int_{0}^{t / 2}(1+t-\tau)^{-n / 4-(k+1) / 2}(1+\tau)^{-n / 2} d \tau \\
& \leq C M(t)^{2}(1+t)^{-n / 4-(k+1) / 2} \int_{0}^{t / 2}(1+\tau)^{-n / 2} d \tau \\
& \leq C M(t)^{2}(1+t)^{-n / 4-k / 2} \eta(t),
\end{aligned}
$$

where

$$
\eta(t)= \begin{cases}1, & n=1 \\ (1+t)^{-1 / 2} \log (2+t), & n=2 \\ (1+t)^{-1 / 2}, & n \geq 3\end{cases}
$$

On the other hand, we have $\left\|\partial_{x}^{k} f(u)\right\|_{L^{2}} \leq C\|u\|_{L^{\infty}}\left\|\partial_{x}^{k} u\right\|_{L^{2}}$ by Lemma 5. Therefore, using (50), we find that $\left\|\partial_{x}^{k} f(u)(\tau)\right\|_{L^{2}} \leq$ $C M(t)^{2}(1+\tau)^{-n / 4-k / 2-n / 2}$. Consequently, we can estimate the term $J_{12}$ as

$$
J_{12} \leq C M(t)^{2} \int_{0}^{t / 2} e^{-c(t-\tau)}(1+\tau)^{-n / 4-k / 2-n / 2} d \tau \leq C M(t)^{2} e^{-c t}
$$

Finally, we estimate the term $J_{2}$ on the time interval $[t / 2, t]$. Applying (42) with $p=2, j=k$, and $l=0$ and using $\left\|\partial_{x}^{k} f(u)(\tau)\right\|_{L^{2}} \leq C M(t)^{2}(1+\tau)^{-n / 4-k / 2-n / 2}$, we obtain

$$
\begin{aligned}
J_{2} \leq & C \int_{t / 2}^{t}(1+t-\tau)^{-1 / 2}\left\|\partial_{x}^{k} f(u)(\tau)\right\|_{L^{2}} d \tau \\
& +C \int_{t / 2}^{t} e^{-c(t-\tau)}\left\|\partial_{x}^{k} f(u)(\tau)\right\|_{L^{2}} d \tau \\
\leq & C M(t)^{2} \int_{t / 2}^{t}(1+t-\tau)^{-1 / 2}(1+\tau)^{-n / 4-k / 2-n / 2} d \tau \\
\leq & C M(t)^{2}(1+t)^{-n / 4-k / 2-(n-1) / 2} .
\end{aligned}
$$

Thus we have shown that

$$
J \leq C M(t)^{2}(1+t)^{-n / 4-k / 2} \eta(t) .
$$

Substituting all these estimates into (51), we obtain

$$
(1+t)^{n / 4+k / 2}\left\|\partial_{x}^{k} u(t)\right\|_{L^{2}} \leq C E_{0}+C M(t)^{2}
$$

for $0 \leq k \leq s+2$. Consequently, we have $M(t) \leq C E_{0}+$ $C M(t)^{2}$, from which we can deduce $M(t) \leq C E_{0}$, provided that $E_{0}$ is suitably small. This proves the decay estimate (47).

In what follows, we prove the decay estimate (48) for the time derivative $u_{t}$. For this purpose, we differentiate (19) with respect to $t$ to obtain

$$
\begin{aligned}
u_{t}(t)= & G_{t}(t) * u_{1}+H_{t}(t) * u_{1} \\
& +\int_{0}^{t} G_{t}(t-\tau) * \Delta f(u)(\tau) d \tau .
\end{aligned}
$$

Applying $\partial_{x}^{k}$ to (61) and taking the $L^{2}$ norm, we have

$$
\begin{aligned}
\left\|\partial_{x}^{k} u_{t}(t)\right\|_{L^{2}} \leq & \left\|\partial_{x}^{k} G_{t}(t) * u_{1}\right\|_{L^{2}}+\left\|\partial_{x}^{k} H_{t}(t) * u_{0}\right\|_{L^{2}} \\
& +\int_{0}^{t}\left\|\partial_{x}^{k} G_{t}(t-\tau) * \Delta f(u)(\tau)\right\|_{L^{2}} d \tau \\
:= & I_{1}^{\prime}+I_{2}^{\prime}+J^{\prime}
\end{aligned}
$$

where $0 \leq k \leq s$. For the term $I_{1}^{\prime}$, we apply (37) with $p=1$, $j=0$, and $l=0$ to get

$$
\begin{aligned}
I_{1}^{\prime} \leq & C(1+t)^{-n / 4-(k+1) / 2}\left\|u_{1}\right\|_{\dot{W}^{-1,1}} \\
& +e^{-c t}\left\|\partial_{x}^{k} u_{1}\right\|_{L^{2}} \leq C E_{0}(1+t)^{-n / 4-(k+1) / 2} .
\end{aligned}
$$

Also, for the term $I_{2}^{\prime}$, applying (38) with $p=1, j=0$, and $l=0$, we have

$$
\begin{aligned}
I_{2}^{\prime} \leq & C(1+t)^{-n / 4-(k+1) / 2}\left\|u_{0}\right\|_{L^{1}} \\
& +e^{-c t}\left\|\partial_{x}^{k+2} u_{0}\right\|_{L^{2}} \leq C E_{0}(1+t)^{-n / 4-(k+1) / 2} .
\end{aligned}
$$

To estimate the nonlinear term $J^{\prime}$, we divide $J_{1}^{\prime}$ as $J^{\prime}=J_{1}^{\prime}+J_{2}^{\prime}$, where $J_{1}^{\prime}$ and $J_{2}^{\prime}$ correspond to the time intervals $[0, t / 2]$ and $[t / 2, t]$, respectively. For the term $J_{1}^{\prime}$, we apply (43) with $p=1$, $j=0$, and $l=0$. This yields

$$
\begin{aligned}
J_{1}^{\prime} \leq & C \int_{0}^{t / 2}(1+t-\tau)^{-n / 4-(k+2) / 2}\|f(u)(\tau)\|_{L^{1}} d \tau \\
& +C \int_{0}^{t / 2} e^{-c(t-\tau)}\left\|\partial_{x}^{k+2} f(u)(\tau)\right\|_{L^{2}} d \tau \\
:=J_{11}^{\prime} & +J_{12}^{\prime} .
\end{aligned}
$$

Since $\|f(u)(\tau)\|_{L^{1}} \leq C M(t)^{2}(1+\tau)^{-n / 2}$ as before, we can estimate the term $J_{11}^{\prime}$ as

$$
\begin{aligned}
J_{11}^{\prime} & \leq C M(t)^{2} \int_{0}^{t / 2}(1+t-\tau)^{-n / 4-(k+2) / 2}(1+\tau)^{-n / 2} d \tau \\
& \leq C M(t)^{2}(1+t)^{-n / 4-(k+1) / 2} \eta(t) .
\end{aligned}
$$


Also, the term $J_{12}^{\prime}$ is estimated similarly as before, and we have $J_{12}^{\prime} \leq C M(t)^{2} e^{-c t}$. Finally, we estimate the term $J_{2}^{\prime}$ by applying (43) with $p=2, j=k+2$, and $l=0$ and obtain

$$
\begin{aligned}
J_{2}^{\prime} \leq & C \int_{t / 2}^{t}\left\|\partial_{x}^{k+2} f(u)(\tau)\right\|_{L^{2}} d \tau \\
& +C \int_{t / 2}^{t} e^{-c(t-\tau)}\left\|\partial_{x}^{k+2} f(u)(\tau)\right\|_{L^{2}} d \tau \\
\leq & C M(t)^{2} \int_{t / 2}^{t}(1+\tau)^{-n / 4-(k+2) / 2-n / 2} d \tau \\
\leq & C M(t)^{2}(1+t)^{-n / 4-(k+1) / 2-(n-1) / 2},
\end{aligned}
$$

where we used the estimate $\left\|\partial_{x}^{k+2} f(u)(\tau)\right\|_{L^{2}} \leq$ $C M(t)^{2}(1+\tau)^{-n / 4-(k+2) / 2-n / 2}$. Consequently we have shown that

$$
J^{\prime} \leq C M(t)^{2}(1+t)^{-n / 4-(k+1) / 2} \eta(t) .
$$

Substituting all these estimates together with the previous estimate $M(t) \leq C E_{0}$ into (62), we arrive at the desired estimate (48) for $0 \leq k \leq s$. This completes the proof of Theorem 6.

The previous proof of Theorem 6 shows that when $n \geq 2$, the solution $u$ to the integral equation (19) is asymptotic to the linear solution $u_{L}$ given by the formula $u_{L}(t):=G(t) * u_{1}+$ $H(t) * u_{0}$ in (18) as $t \rightarrow \infty$. This result is stated as follows.

Corollary 7. Let $n \geq 2$ and assume the same conditions of Theorem 6. Then the solution $u$ of the problem (1), (2), which is constructed in Theorem 6, can be approximated by the solution $u_{L}$ to the linearized problem (8), (2) as $t \rightarrow \infty$. More precisely, one has the following asymptotic relations:

$$
\begin{gathered}
\left\|\partial_{x}^{k}\left(u-u_{L}\right)(t)\right\|_{L^{2}} \leq C E_{0}^{2}(1+t)^{-n / 4-k / 2} \eta(t), \\
\left\|\partial_{x}^{k}\left(u-u_{L}\right)_{t}(t)\right\|_{L^{2}} \leq C E_{0}^{2}(1+t)^{-n / 4-(k+1) / 2} \eta(t)
\end{gathered}
$$

for $0 \leq k \leq s+2$ and $0 \leq k \leq s$, respectively, where $u_{L}(t):=$ $G(t) * u_{1}+H(t) * u_{0}$ is the linear solution and $\eta(t)$ is defined in (56).

\section{Asymptotic Linear Profile}

In the previous section, we have shown that the solution $u$ to the problem (1), (2) can be approximated by the linear solution $u_{L}$. The aim of this section is to derive a simpler asymptotic profile of the linear solution $u_{L}$.

In Section 2, we derive the solution formula $u_{L}(t)=G(t) *$ $u_{1}+H(t) * u_{0}$ to the problem (8), (9). In the Fourier space, we have $\widehat{u}_{L}(\xi, t)=\widehat{G}(\xi, t) \widehat{u}_{1}(\xi)+\widehat{H}(\xi, t) \widehat{u}_{0}(\xi)$, where $\widehat{G}(\xi, t)$ and $\widehat{H}(\xi, t)$ are given explicitly in (14) and (15), respectively. First we give the asymptotic expansions of $\widehat{G}(\xi, t)$ and $\widehat{H}(\xi, t)$ for $\xi \rightarrow 0$. Applying the Taylor expansion to (12), we see that

$$
\begin{aligned}
\lambda_{ \pm}(\xi) & =-b|\xi|^{2} \pm i|\xi| \sqrt{1+\left(\alpha-b^{2}\right)|\xi|^{2}} \\
& =-b|\xi|^{2} \pm i|\xi|\left(1+\frac{1}{2}\left(\alpha-b^{2}\right)|\xi|^{2}+O\left(|\xi|^{4}\right)\right) \\
& = \pm i|\xi|-b|\xi|^{2}+O\left(|\xi|^{3}\right), \\
\frac{1}{\lambda_{+}-\lambda_{-}} & =\frac{1}{2 i|\xi| \sqrt{1+\left(\alpha-b^{2}\right)|\xi|^{2}}} \\
& =\frac{1}{2 i|\xi|}\left\{1-\frac{1}{2}\left(\alpha-b^{2}\right)|\xi|^{2}+O\left(\xi^{4}\right)\right\} .
\end{aligned}
$$

Inserting the previous equalities into (14) and (15), respectively, we arrive at

$$
\begin{aligned}
\widehat{G}(\xi, t)= & \frac{e^{\lambda_{+} t}-e^{\lambda_{-} t}}{\lambda_{+}-\lambda_{-}} \\
= & \frac{1}{2 i|\xi|}\left\{e^{-b|\xi|^{2} t}\left(e^{|\xi| t i}-e^{-|\xi| t i}\right)\right. \\
& \left.\quad+e^{-b|\xi|^{2} t}\left(O(|\xi|)+O\left(|\xi|^{3} t\right)\right)\right\}, \\
\widehat{H}(\xi, t)= & \frac{\lambda_{+} e^{\lambda_{-} t}-\lambda_{-} e^{\lambda_{+} t}}{\lambda_{+}-\lambda_{-}} \\
= & \frac{1}{2} e^{-b|\xi|^{2} t}\left(e^{|\xi| t i}+e^{-|\xi| t i}\right) \\
& +e^{-b|\xi|^{2} t}\left\{O(|\xi|)+O\left(|\xi|^{3} t\right)\right\}
\end{aligned}
$$

for $\xi \rightarrow 0$. Taking

$$
\begin{gathered}
\widehat{G}_{0}(\xi, t)=\frac{1}{2 i|\xi|} e^{-b|\xi|^{2} t}\left(e^{i|\xi| t}-e^{-i|\xi| t}\right) \\
\widehat{H}_{0}(\xi, t)=\frac{1}{2} e^{-b|\xi|^{2} t}\left(e^{i|\xi| t}+e^{-i|\xi| t}\right)
\end{gathered}
$$

Then we find that

$$
\begin{gathered}
\left|\left(\widehat{G}-\widehat{G}_{0}\right)(\xi, t)\right| \leq C e^{-c|\xi|^{2} t}, \\
\left|\left(\widehat{H}-\widehat{H}_{0}\right)(\xi, t)\right| \leq C|\xi| e^{-c|\xi|^{2} t}
\end{gathered}
$$

for $|\xi| \leq r_{0}$, where $r_{0}$ is a small positive constant.

We now define $\bar{u}_{L}$ by

$$
\bar{u}_{L}(t)=G_{0}(t) * u_{1}+H_{0}(t) * u_{0} .
$$

Then $\bar{u}_{L}$ gives a asymptotic profile of the linear solution $u_{L}$. In fact we have the following. 
Theorem $8(n \geq 1$ and $s \geq 0)$. Assume that $u_{0} \in H^{s+2} \cap L^{1}$ and $u_{1} \in H^{s} \cap \dot{W}^{-1,1}$, and put $E_{0}=\left\|u_{0}\right\|_{L^{1}}+\left\|u_{1}\right\|_{\dot{W}^{-1,1}}+\left\|u_{0}\right\|_{H^{s+2}}+$ $\left\|u_{1}\right\|_{H^{s}}$. Let $u_{L}$ be the linear solution, and let $\bar{u}_{L}$ be defined by (74). Then one has

$$
\left\|\partial_{x}^{k}\left(u_{L}-\bar{u}_{L}\right)(t)\right\|_{L^{2}} \leq C E_{0}(1+t)^{-n / 4-(k+1) / 2}
$$

for $0 \leq k \leq s+2$.

Proof. Note that $\left(u_{L}-\bar{u}_{L}\right)(t)=\left(G-G_{0}\right)(t) * u_{1}+\left(H-H_{0}\right)(t) *$ $u_{0}$, so for the proof of (75), it suffices to show the following estimates:

$$
\begin{aligned}
\left\|\partial_{x}^{k}\left(G-G_{0}\right)(t) * u_{1}\right\|_{L^{2}} & \\
\leq & C(1+t)^{-(n / 2)(1 / p-1 / 2)-(k+1-j) / 2}\left\|\partial_{x}^{j} u_{1}\right\|_{\dot{W}^{-1, p}} \\
& +C e^{-c t}\left\|\partial_{x}^{k+l-2} u_{1}\right\|_{L^{2}}, \\
\left\|\partial_{x}^{k}\left(H-H_{0}\right)(t) * u_{0}\right\|_{L^{2}} & \\
\leq & C(1+t)^{-(n / 2)(1 / p-1 / 2)-(k+1-j) / 2}\left\|\partial_{x}^{j} u_{0}\right\|_{L^{p}} \\
& +C e^{-c t}\left\|\partial_{x}^{k+l} u_{0}\right\|_{L^{2}},
\end{aligned}
$$

where $1 \leq p \leq 2$ and $k, j$, and $l$ are nonnegative integers such that $0 \leq j \leq k+1$; we assumed that $k+l-2 \geq 0$ in the first estimate. These estimates can be proved similarly as in the proof of Lemma 3 by using (73) for $|\xi| \leq r_{0}$ and (31), (32), and (72) for $|\xi| \geq r_{0}$. Here we omit the details.

\section{Acknowledgment}

This work was supported in part by the NNSF of China (Grant no. 11101144).

\section{References}

[1] J. Boussinesq, "Théorie des ondes et des remous qui se propagent le long dùn canal rectangu-laire horizontal, en communiquant au liquide contenu dans ce canal des vitesses sensiblement pareilles de la surface au fond," Journal de Mathématiques Pures et Appliquées, vol. 17, pp. 55-108, 1872.

[2] V. Varlamov, "On the Cauchy problem for the damped Boussinesq equation," Differential and Integral Equations, vol. 9, no. 3, pp. 619-634, 1996.

[3] V. Varlamov, "Existence and uniqueness of a solution to the Cauchy problem for the damped Boussinesq equation," Mathematical Methods in the Applied Sciences, vol. 19, no. 8, pp. 639649, 1996.

[4] V. V. Varlamov, "Asymptotic behavior of solutions of the damped Boussinesq equation in two space dimensions," International Journal of Mathematics and Mathematical Sciences, vol. 22, no. 1, pp. 131-145, 1999.

[5] W.-R. Dai and D.-X. Kong, "Global existence and asymptotic behavior of classical solutions of quasilinear hyperbolic systems with linearly degenerate characteristic fields," Journal of Differential Equations, vol. 235, no. 1, pp. 127-165, 2007.

[6] D.-X. Kong and T. Yang, "Asymptotic behavior of global classical solutions of quasilinear hyperbolic systems," Communications in Partial Differential Equations, vol. 28, no. 5-6, pp. 1203-1220, 2003.

[7] M. Nakao and K. Ono, "Existence of global solutions to the Cauchy problem for the semilinear dissipative wave equations," Mathematische Zeitschrift, vol. 214, no. 2, pp. 325-342, 1993.

[8] K. Nishihara, " $L^{p}-L^{q}$ estimates of solutions to the damped wave equation in 3-dimensional space and their application," Mathematische Zeitschrift, vol. 244, no. 3, pp. 631-649, 2003.

[9] K. Ono, "Global existence and asymptotic behavior of small solutions for semilinear dissipative wave equations," Discrete and Continuous Dynamical Systems A, vol. 9, no. 3, pp. 651-662, 2003.

[10] Z. Yang, "Longtime behavior of the Kirchhoff type equation with strong damping on $\mathbb{R}^{n}$," Journal of Differential Equations, vol. 242, no. 2, pp. 269-286, 2007.

[11] Y. Sugitani and S. Kawashima, "Decay estimates of solutions to a semilinear dissipative plate equation," Journal of Hyperbolic Differential Equations, vol. 7, no. 3, pp. 471-501, 2010.

[12] Y.-X. Wang and Z. Wei, "Global existence and asymptotic behavior of solutions to Cahn-Hilliard equation with inertial term," International Journal of Mathematics, vol. 23, no. 9, p. 1250087, 14, 2012.

[13] Y. Wang, "Existence and asymptotic behavior of solutions to the generalized damped Boussinesq equation," Electronic Journal of Differential Equations, no. 96, 11 pages, 2012.

[14] Y.-Z. Wang, F. Liu, and Y. Zhang, "Global existence and asymptotic behavior of solutions for a semi-linear wave equation," Journal of Mathematical Analysis and Applications, vol. 385, no. 2, pp. 836-853, 2012.

[15] Y.-Z. Wang and Y.-X. Wang, "Global existence and asymptotic behavior of solutions to a nonlinear wave equation of fourthorder," Journal of Mathematical Physics, vol. 53, no. 1, p. 013512, 13, 2012.

[16] S. Kawashima and Y.Z. Wang, "Global existenceand asymptotic behavior of solutions to the generalized cubic double dispersion equation," Analysis and Applications. In press.

[17] S. Wang and H. Xu, "On the asymptotic behavior of solution for the generalized IBq equation with hydrodynamical damped term," Journal of Differential Equations, vol. 252, no. 7, pp. 42434258, 2012.

[18] T. T. Li and Y. M. Chen, Nonlinear Evolution Equations, Scientific Press, 1989, (Chinese).

[19] S. Zheng, Nonlinear Evolution Equations, vol. 133 of Monographs and Surveys in Pure and Applied Mathematics, Chapman \& Hall, 2004. 


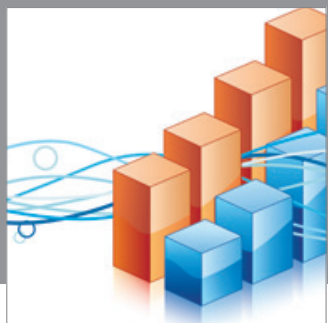

Advances in

Operations Research

mansans

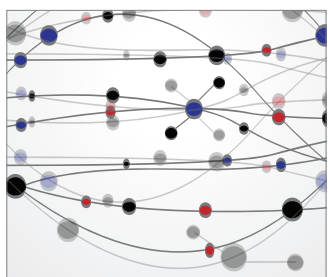

The Scientific World Journal
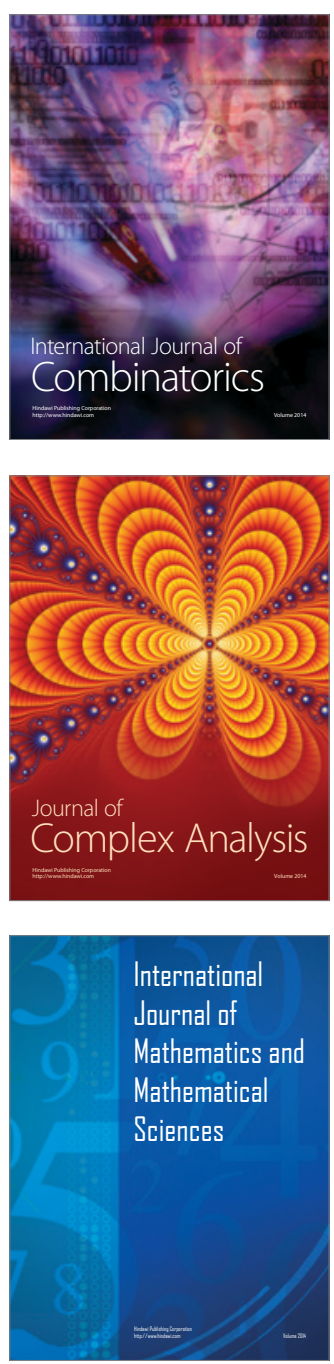
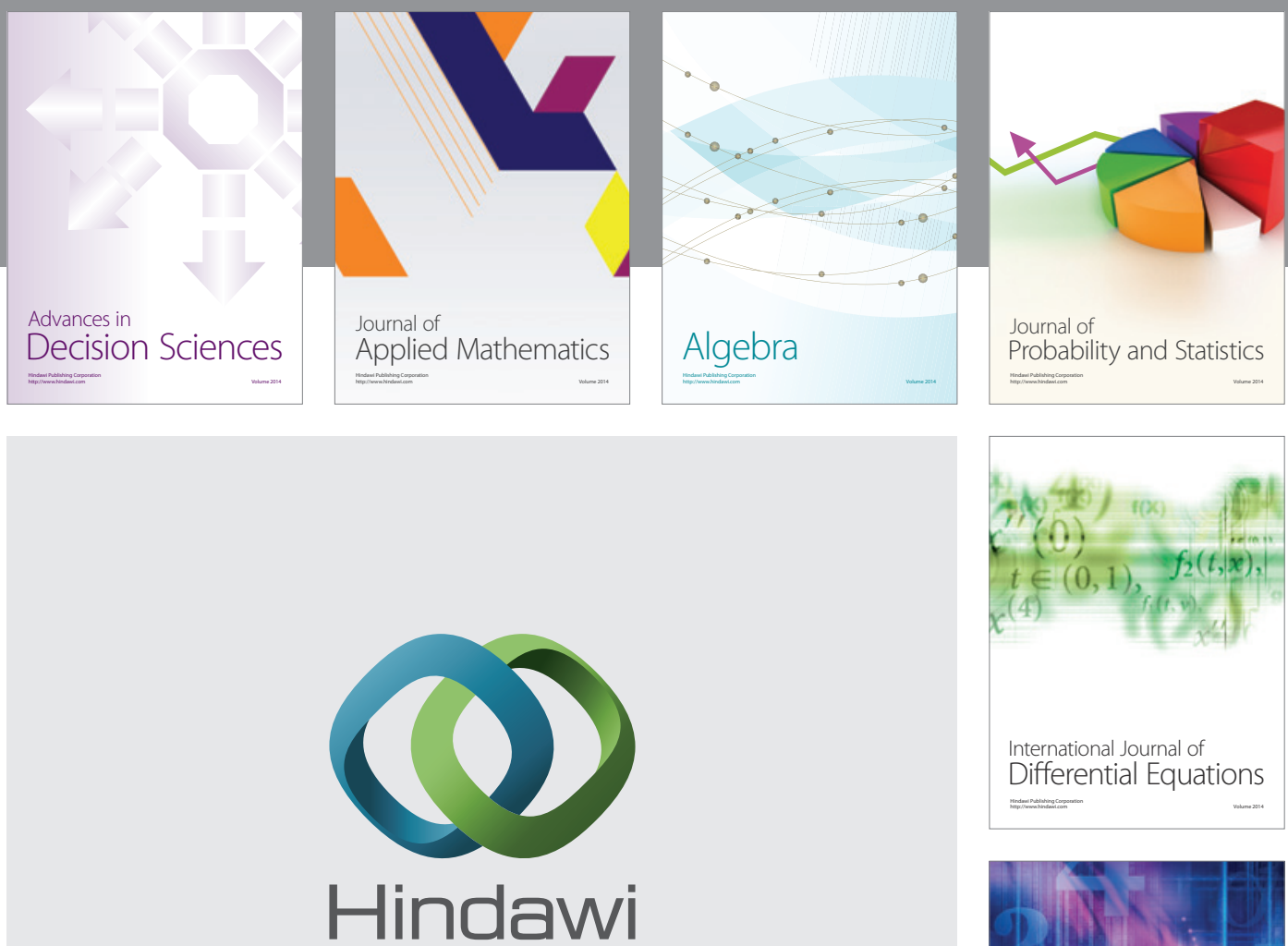

Submit your manuscripts at http://www.hindawi.com
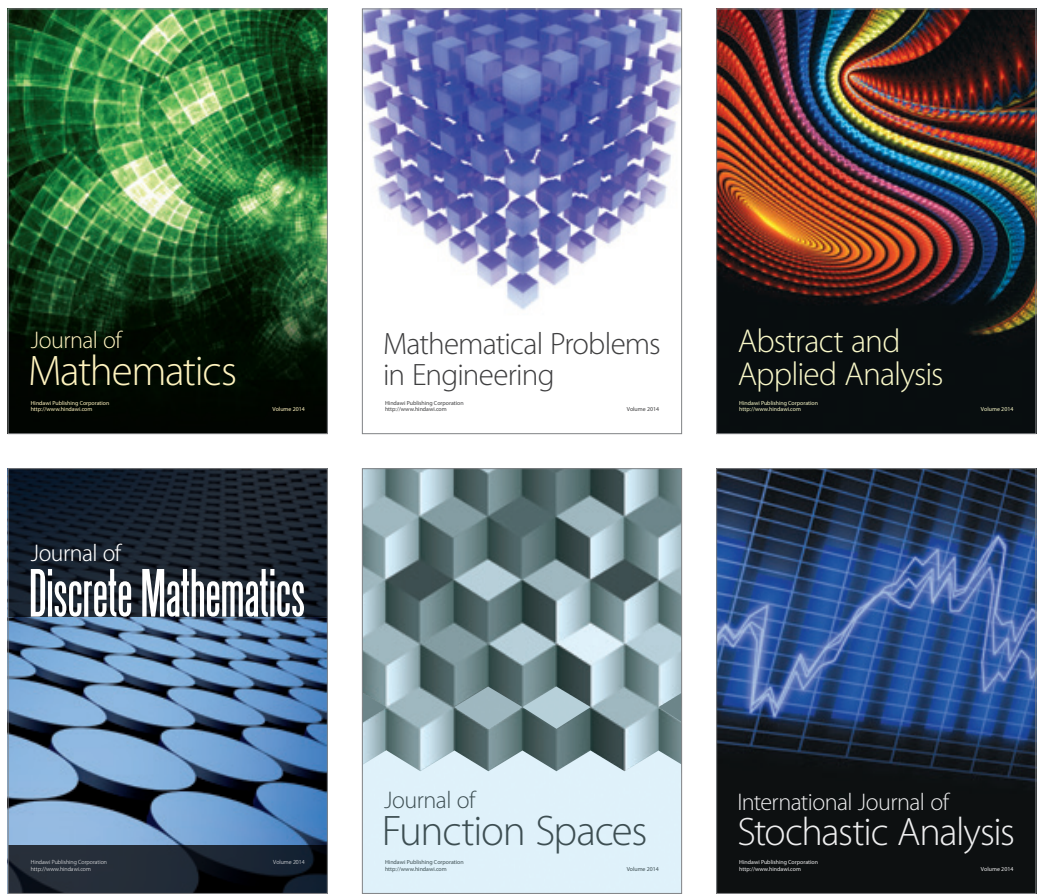

Journal of

Function Spaces

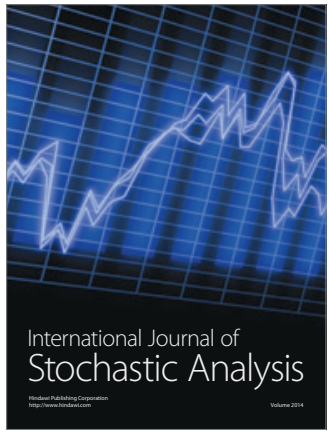

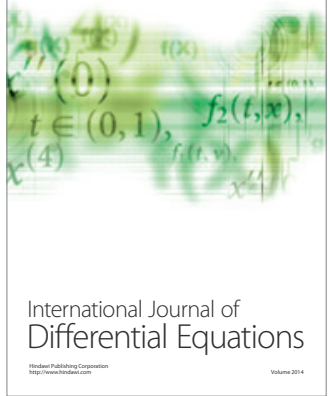
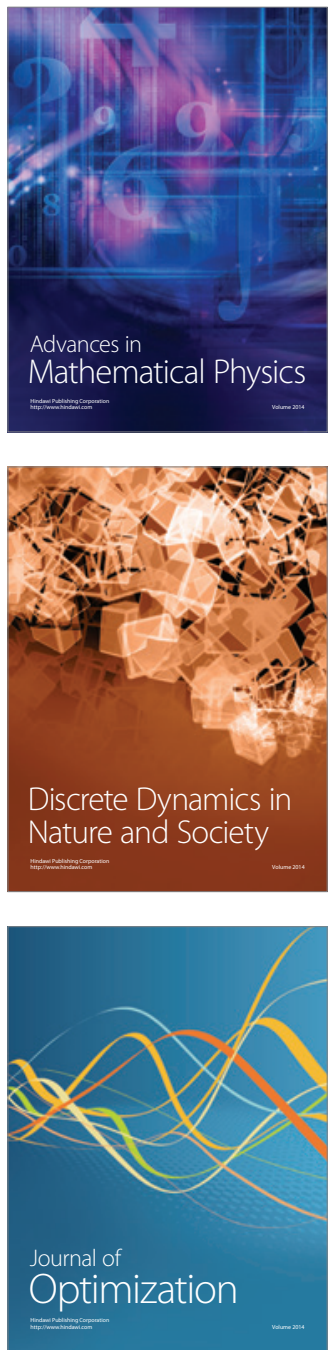